\title{
Conhecimento de enfermeiros sobre higienização e acondicionamento de próteses dentárias removíveis de pacientes em internação hospitalar e fatores associados
}

\author{
Nurses' knowledge about hygienization and packaging of removable dental prosthesis of patients in \\ hospital admission and associated factors
}

Conocimiento de las enfermeras sobre higienización y embalaje de prótesis dental removible de pacientes en admisión hospitalaria y factores asociados

Recebido: 20/07/2021 | Revisado: 27/07/2021 | Aceito: 13/08/2021 | Publicado: 16/08/2021

\begin{abstract}
Resumo
Objetivou-se avaliar o conhecimento da equipe de enfermagem do Hospital São Vicente de Paulo (HSVP) em João Pessoa, PB, sobre higienização e acondicionamento de próteses dentárias removíveis de pacientes hospitalizados, e fatores associados. Trata-se de um estudo observacional transversal quantitativo, realizado com abordagem pelo método dedutivo e análise através de frequências absolutas e percentuais. O universo foi composto por todos os enfermeiros que trabalham no HSVP $(n=36)$, dos quais 34 compuseram a amostra deste estudo. A coleta de dados só iniciou após aprovação da pesquisa pelo Comitê de Ética em Pesquisa do Centro Universitário UNIESP (No 4.315.662), sendo realizada por meio da aplicação de um questionário específico. Foi observado que a maioria das enfermeiras $(91,20 \%$, $\mathrm{n}=31$ ) não realizava a higienização das próteses de seus pacientes, nem receberam nenhum tipo de treinamento relacionado aos cuidados bucais, tampouco, com as próteses dentárias. Identificou-se que apenas $23,57 \%(\mathrm{n}=8)$ das enfermeiras acondicionavam as próteses dentárias de maneira adequada. A respeito da temática do estudo, 97,10\% (n= 33) acham interessante receber treinamento. Foi observada associação entre não ter recebido treinamento sobre higiene e acondicionamento de próteses dentárias e indicar de forma equivocada materiais para higiene das próteses $(\mathrm{RR}=10,40$; $\mathrm{IC}=0,785-137,83 ; \mathrm{p}=0,039)$. Conclui-se que a falta de conhecimento e treinamento da equipe de enfermagem do HSVP refletiu negativamente na realização da higienização e acondicionamento de próteses dentárias de pacientes hospitalizados.
\end{abstract}

Palavras-chave: Equipe hospitalar de odontologia; Pacientes internados; Prótese dentária.

\begin{abstract}
The objective was to evaluate the knowledge of the nursing staff of Hospital São Vicente de Paulo (HSVP) in João Pessoa, PB, on hygiene and packaging of removable dental prostheses for hospitalized patients, and associated factors. This is a cross sectional observational quantitative study, carried out using the deductive method and analysis using absolute and percentage frequencies. The universe was composed of all nurses who work at HSVP $(n=36)$, of which 34 comprised the sample of this study. Data collection only started after approval of the research by the Research Ethics Committee of the Centro Universitário UNIESP (No. 4,315,662), being carried out through the application of a specific questionnaire. It was observed that most nurses $(91.20 \%, \mathrm{n}=31)$ did not perform the cleaning of their patients' prostheses, nor did they receive any type of training related to oral care, either, with dental prostheses. It was identified that only $23.57 \%(\mathrm{n}=8)$ of the nurses adequately conditioned their dental prostheses. Regarding the theme of the study, $97.10 \%(\mathrm{n}=33)$ found it interesting to receive training. An association was observed between not having received training on hygiene and packaging of dental prostheses and mistakenly indicating materials for hygiene of the prostheses $(\mathrm{RR}=10.40 ; \mathrm{CI}=0.785-137.83 ; \mathrm{p}=0.039)$. It is concluded that the lack of knowledge and training of the HSVP nursing team reflected negatively in the cleaning and conditioning of dental prostheses for hospitalized patients.
\end{abstract}

Keywords: Hospital dentistry team; Hospitalized patients; Dental prosthetics. 


\section{Resumen}

El objetivo de estudio fue evaluar el conocimiento de los enfermeros del Hospital São Vicente de Paulo (HSVP) sobre limpieza y empaque de prótesis dentales removibles en pacientes hospitalizados y factores asociados. Se trata de un estudio observacional transversal cuantitativo, realizado con un enfoque por el método deductivo y análisis mediante frecuencias absolutas y porcentajes. El universo estuvo compuesto por todos los enfermeros que laboran en el HSVP (n = 36), de los cuales 34 conformaron la muestra para este estudio. La recogida de datos sólo se inició tras la aprobación de la investigación por parte del Comité de Ética en Investigación del Centro Universitario UNIESP ( $\mathrm{n}^{\circ}$ 4.315.662), realizándose mediante la aplicación de un cuestionario específico. Se observó que la mayoría de enfermeras $(91,20 \%$, $\mathrm{n}=31$ ) no realizaba la limpieza de las dentaduras postizas de sus pacientes, ni recibían ningún tipo de formación relacionada con el cuidado bucal, ni con las prótesis dentales. Se encontró que solo el $23,57 \%(n=8)$ de las enfermeras empaquetaron adecuadamente sus prótesis dentales. En cuanto a la temática de estudio, al 97,10\% $(n=33)$ les pareció interesante recibir formación. Se observó asociación entre no haber recibido formación en higiene y embalaje de prótesis dentales e indicar incorrectamente los materiales para la higiene de las prótesis $(\mathrm{RR}=10,40 ; \mathrm{IC}=0,785-137,83 ; \mathrm{p}=$ 0,039). Se concluye que la falta de conocimiento y formación de los enfermeros del HSVP repercutió negativamente en la realización de limpieza y envasado de prótesis dentales en pacientes hospitalizados.

Palabras clave: Equipo de odontología hospitalaria; Pacientes hospitalizados; Prótesis dental.

\section{Introdução}

A Odontologia Hospitalar vem ganhando visibilidade no cenário multiprofissional, pela atuação conjunta nas equipes hospitalares, contribuindo com a redução dos índices de morbidade e mortalidade decorrentes da disseminação de problemas bucais para outros órgãos (Amaral et al., 2018; Aranega et al., 2012). A atuação do cirurgião-dentista (CD) no ambiente hospitalar não se delimita exclusivamente à atenção em traumatologia bucomaxilofacial (Aranega et al., 2012), mas também pelo controle da dor, sangramento e infecções da cavidade bucal (Rocha \& Ferreira, 2014). Com isso, a desorganização do biofilme bucal contribui significativamente para a diminuição de infecções respiratórias, redução de óbitos e de custos hospitalares (Coker, Ploeg, Kaasalainen \& Carter, 2016; Rocha \& Ferreira, 2014).

No ambiente hospitalar, os enfermeiros são os profissionais que têm o dever de prestar assistência aos pacientes e à família no processo do nascer, viver, morrer e luto (Conselho Regional de enfermagem [COREN], 2009), tornando-se peça fundamental na garantia da saúde bucal dos pacientes internados (Blum, Silva, Baeder \& Bona, 2018). A atuação da enfermagem na terapia intensiva, necessita passar por treinamentos operacionais para a realização da higienização da cavidade bucal, promovido pelo CD da equipe multiprofissional, com a finalidade de garantir uma maior aproximação entre o conhecimento destas duas áreas e garantia do bem-estar bucal e sistêmico (Blum et al., 2018; Mattevi, Mello, Scannapieco \& Carcereri, 2018).

No entanto, a falta de conhecimento sobre higiene bucal, a falta de material necessário à higiene bucal, sobrecarga de trabalho e a subestimação da importância dos cuidados bucais, configuram-se como barreiras encontradas por enfermeiros que atuam em ambiente hospitalar (Van noort et al., 2019). Como também, quando os enfermeiros se deparam com pacientes usuários de próteses dentárias (Fonseca et al., 2019), as quais requerem todo um cuidado especial, pois o acúmulo de microrganismos nas próteses dentárias representa um fator de risco para o desenvolvimento de inúmeras doenças bucais e sistêmicas (Baba, Sato, Owada \& Minakuchi, 2018).

Uma gama de métodos são conhecidos e discutidos na higienização de próteses removíveis, sendo eles: utilização de escovas com cerdas duras ou específicas para remoção mecânica, utilização de comprimidos de limpeza, creme dental convencional não abrasivo, água pura/doce, sabão e água, creme dental especial para dentaduras, limpador de próteses em líquido, limpador de próteses em espuma, detergente, enxaguante bucal, bicarbonato de sódio, vinagre, água salgada, alvejante, entre outros (Axe, Varghese, Bosma, Kitson \& Bradshaw, 2015). Outro método bastante eficaz na desinfecção de próteses totais removíveis é o método de irradiação por micro-ondas, que reduz significativamente o número de microrganismos presentes na prótese (Dantas, Consani, Sardi \& Mesquita, 2014). No entanto, o método mais utilizado e indicado é a escovação, que promove remoção considerável no percentual de extensão do biofilme presente na prótese (Papadiochou \& Polyzois, 2017), sendo 
imprescindível a utilização de substâncias químicas, como os peróxidos alcalinos, que removem manchas suaves e possuem efeito antibacteriano e fungicida e não danificam os componentes constituintes das próteses (metais e resina acrílica) (Gonçalves, Silva Neto, Bonan, Carlo \& Batista, 2011). E, durante o sono, é indicado que a prótese seja removida e acondicionada num reservatório úmido exclusivo, visando reduzir a proliferação de microrganismos patogênicos, bem como, evitar distorções a prótese (Fonseca et al., 2019).

Quando internados, na maioria das vezes, o acondicionamento e higiene das próteses ficam sob responsabilidade da equipe de saúde, embora a perda desses dispositivos em ambiente hospitalar ainda ser muito comum, devido à falta de acondicionamento adequado por parte da equipe de enfermagem (Axe et al., 2015; Mann \& Doshi, 2017). Para tanto, se faz necessária intervenção do CD no treinamento da equipe, visando obter melhores resultados no acondicionamento e orientação de higienização das próteses (Fonseca et al., 2019).

Diante do exposto, percebe-se a importância da realização de estudos sobre a higiene e acondicionamento de próteses dentárias, de indivíduos hospitalizados, pela equipe de enfermagem. Estudos desta natureza impulsionam intervenções educativas, a fim de treinar enfermeiros no controle do biofilme e correto acondicionamento de próteses dentárias removíveis, tendo em vista que a mal higiene e o acondicionamento inadequados resultarão em sérios prejuízos para os pacientes internados em hospitais. Além disso, estes dispositivos são essenciais para melhoria da qualidade de vida e recuperação desta população. Com isso, buscou-se avaliar o conhecimento de enfermeiros do Hospital São Vicente de Paulo sobre a higienização e acondicionamento de próteses dentárias removíveis de pacientes hospitalizados.

\section{Metodologia}

O estudo trata-se de uma pesquisa observacional, transversal, quantitativo, realizado com abordagem pelo método dedutivo e análise através de frequências absolutas e percentuais. A técnica de observação foi indireta e extensiva, utilizando os dados coletados por um instrumento específico do presente projeto.

Esse estudo foi realizado no Hospital São Vicente de Paulo, situado na Avenida João Machado, 1234, Centro, João Pessoa - PB (CEP: 58013-522). O hospital conta com 125 leitos nas Unidades de Internação, 12 leitos no Centro de Terapia Intensiva, 9 leitos de Unidades Intermediárias, 6 salas de Blocos Cirúrgicos, 2 salas de Recuperação, 6 salas de para Serviços de Consultas e 5 camas de observação no Serviço de Emergência. É referência de alta e média complexidade em neurologia, nefrologia, oncologia e angiologia. Os serviços disponíveis envolvem procedimentos eletivos e de urgência/emergência em clínica médica e clínica cirúrgica.

A amostra foi composta por enfermeiros que formam o quadro de funcionários do Hospital São Vicente de Paulo. Sendo abordados todos os profissionais cadastrados no conselho de classe da Enfermagem, informados sobre a realização da pesquisa e convidados a participar respondendo um questionário. A seleção dos participantes foi realizada por conveniência. Os que desejaram, de livre e espontânea vontade, participar da pesquisa, assinaram o Termo de Consentimento Livre e Esclarecido TCLE, declarando estar de acordo com os critérios estabelecidos para análise das respostas.

Para serem incluídos no estudo, os enfermeiros deveriam estar regularmente inscritos no Conselho Regional de Enfermagem da Paraíba, contratados há mais de 1 mês para prestação de serviço no Hospital São Vicente de Paulo e que atuassem nas alas de internação/atendimento aos pacientes (enfermaria cardiovascular, enfermaria oncológica, enfermaria nefrológica (cuidados a pacientes renais crônicos), bloco cirúrgico, urgência, UTI) e coordenação. Não foram incluídos questionários rasurados ou incompletos e enfermeiros que solicitassem a exclusão de suas respostas, após a assinatura do TCLE.

Os dados foram coletados por meio de um questionário criado pelo pesquisador responsável exclusivamente para realização desta pesquisa. $\mathrm{O}$ instrumento é composto, inicialmente, por perguntas referentes à caracterização da amostra (sexo, 
idade e local de atuação) e, em seguida, por 14 perguntas objetivas sobre a higienização e acondicionamento de próteses dentárias removíveis. As perguntas vão desde a importância da realização de treinamentos da equipe de enfermagem para tal fim, até a realização de procedimentos de higiene e acondicionamento desses aparelhos. Além disso, pergunta-se sobre fatores associados ao conhecimento e segurança para realização dessas atividades.

Os enfermeiros foram convidados individualmente a participar da pesquisa. Foram orientados sobre a finalidade do estudo, os que aceitaram participar, receberam duas cópias do TCLE contendo as informações do estudo (uma para o sujeito e outra devolvida ao responsável pela coleta de dados). Após a assinatura do termo, foram convidados a um local reservado, a fim de estabelecer a confidencialidade dos dados. Em seguida, os profissionais receberam o questionário e responderam às perguntas no tempo necessário.O instrumento contendo as respostas dos enfermeiros foi armazenado em envelopes não identificados e guardados até o final do prazo estipulado para a coleta das informações. Após o prazo, os dados foram passados para um software, onde foram analisados.

Os dados obtidos foram tabulados no programa Excel e analisados por meio de estatística descritiva e inferencial, com auxílio do software SPSS v.22.0. Utilizou-se os testes Qui-Quadrado de Pearson e Exato de Fisher para verificar associação entre variáveis. As variáveis relacionadas ao material indicado aos pacientes para higiene das próteses e o local de acondicionamento das próteses no ambiente hospitalar foram dicotomizadas em "Respostas corretas" e "Respostas erradas" (Quadro 1). Foram levados em consideração estudos previamente publicados para a categorização das duas variáveis (Cakan et al., 2015; Papadiochou, Polyzois, 2017; Fonseca et al., 2019)

Quadro 1 - Categorização das variáveis relacionadas aos materiais indicados para higiene das próteses e a forma de acondicionamento desses dispositivos. João Pessoa, PB, Brasil, 2021.

\begin{tabular}{|c|c|c|}
\hline VARIÁVEIS & RESPOSTAS CORRETAS & RESPOSTAS ERRADAS \\
\hline $\begin{array}{l}\text { Materiais indicados para } \\
\text { higiene de próteses }\end{array}$ & $\begin{array}{l}\text { Associação entre o método mecânico e } \\
\text { químico (Cakan et al., 2015; Papadiochou, } \\
\text { Polyzois, 2017). } \\
\text { - Escova + hipoclorito; } \\
\text { - Escova + enxaguante; } \\
\text { - Escova + sabão; } \\
\text { - Escova + hipoclorito + enxaguante; } \\
\text { - Escova + sabão + enxaguante. }\end{array}$ & $\begin{array}{l}\text { Métodos isolados ou associados a creme dental. } \\
\text { - Escova; } \\
\text { - } \text { Creme dental; } \\
\text { - Sabão; } \\
\text { - } \text { Hastilhas efervescentes; } \\
\text { - Escova + creme dental; } \\
\text { - Escova + creme dental + enxaguante; } \\
\text { - Escova + creme dental + sabão; } \\
\text { - Escova + creme dental + hipoclorito. }\end{array}$ \\
\hline $\begin{array}{l}\text { Local de acondicionamento } \\
\text { das próteses dentárias no } \\
\text { ambiente hospitalar }\end{array}$ & $\begin{array}{l}\text { Recipiente úmido, fechado e etiquetado com } \\
\text { os demais pertences (Fonseca et al., 2019). } \\
\text { - Sacola plástica úmida fechada } \\
\text { etiquetada com os demais pertences. }\end{array}$ & $\begin{array}{l}\text { Locais diversos que favorecem a mudança estrutural e } \\
\text { perda dos dispositivos no ambiente hospitalar. } \\
\text { - Envolta em uma gaze; } \\
\text { - Envolta em um guardanapo; } \\
\text { - Copo com água; } \\
\text { - Copo com água e hipoclorito; } \\
\text { - Entrega à família; } \\
\text { - Sacola plástica; } \\
\text { - Não guarda. }\end{array}$ \\
\hline
\end{tabular}

Fonte: Autores (2021).

Foi adotado um nível de significância de 5\%, intervalo de confiança de $95 \%$ e poder estatístico de no mínimo $80 \%$.

Os procedimentos para a realização desta pesquisa respeitaram as diretrizes e normas que regulamentam as pesquisas envolvendo seres humanos, aprovadas pela Resolução número 466/12 do Conselho Nacional de Saúde. O presente trabalho foi aprovado pelo ao Comitê de Ética em Pesquisa do Centro Universitário UNIESP. Número do parecer: 4.315.662. 


\section{Resultados}

Dos 36 enfermeiros que trabalham no hospital são vicente de paulo, 34 participaram da pesquisa, onde 100\% (n=34) dos participantes são biologicamente do sexo feminino, com média de 37,32 ( $\pm 9,31)$ anos de idade (Tabela 1).

As enfermeiras atuavam em diversos setores do hospital, como: coordenação $(11,8 \%, n=4)$, urgência $(14,7 \%, n=5)$, bloco cirúrgico $(14,7 \%, n=5)$, uti $(11,8 \%, n=4)$, e em alas destinadas aos cuidados de pacientes com doença renal crônica (11,8\%, $\mathrm{n}=4)$, cardiovascular $(11,8 \%, \mathrm{n}=4)$ e oncológicos $(23,5 \%, \mathrm{n}=8)$.

Observou-se que 88,20\% das enfermeiras $(n=30)$ relataram ter atendido pacientes usuários de próteses dentárias e, diante desta situação, 41,10\% (n=15) afirmaram que removem e entregam as próteses aos familiares, a fim de que esses se responsabilizassem pelo acondicionamento.

Foi identificado que $91,20 \%$ das enfermeiras $(n=31)$ não receberam nenhum tipo de treinamento educativo para realização e/ou orientação de higiene ou acondicionamento de próteses dentárias removíveis. Essa falta de conhecimento refletiu diretamente no interesse pela aquisição de conhecimentos a respeito desta temática, onde 97,10\% (n=33) demostraram interesse em receber informações sobre orientações de higiene e acondicionamento de próteses dentárias removíveis.

Observou-se que $8,80 \%$ das enfermeiras $(n=3)$ realizavam a higienização das próteses de seus pacientes, e os materiais utilizados eram escova de dente $(2,9 \%, \mathrm{n}=1)$, gaze $(2,9 \%, \mathrm{n}=1)$ ou hipoclorito de sódio $(2,9 \%, \mathrm{n}=1)$.

Quando questionadas sobre quais materiais elas indicavam para realização da higiene das próteses dentárias, a indicação de creme dental, escova e enxaguante bucal prevaleceu entre as respostas $(23,5 \%, \mathrm{n}=8)$.

Sobre o acondicionamento das próteses de pacientes internos, a maioria das enfermeiras $(32,40 \%, \mathrm{n}=11)$ afirmaram que guardavam as próteses em uma sacola plástica identificada com o nome do paciente.

Com relação à segurança que as enfermeiras sentiam para realizar a higiene das próteses dos pacientes, 61,80\% afirmaram não se sentir seguras para realizar esta função. Essa insegurança também foi observada quando questionadas sobre sentir segurança em ensinar o paciente a realizar sua própria higienização das próteses, 70,60\% (n=24) não se sentiam seguras para passar essa informação.

A maioria das enfermeiras $(70,60 \%, \mathrm{n}=24)$ afirmaram não possuir uma rotina exaustiva de trabalho no hospital.

Observou-se que $97,10 \%(n=33)$ das enfermeiras, com exceção de uma, acham interessante a criação de um protocolo para a higiene e acondicionamento de próteses dentárias no hospital em que trabalhavam.

Tabela 1. Dados descritivos de enfermeiras questionadas sobre higiene e acondicionamento de próteses dentárias removíveis em ambiente hospitalar. João Pessoa-PB, Brasil, 2021. n=34.

\begin{tabular}{|c|c|c|c|c|}
\hline Variáveis & Categorias & $\begin{array}{c}\text { Frequência } \\
\text { absoluta }\end{array}$ & $\%$ & Total \\
\hline \multirow{2}{*}{ Sexo } & Feminino & 34 & 100,00 & \multirow[t]{2}{*}{34} \\
\hline & Masculino & 0 & 0,00 & \\
\hline Idade - média ( $\pm \mathrm{DP})$ & \multicolumn{2}{|c|}{$37,32( \pm 9,31)$} & & \multirow{8}{*}{34} \\
\hline \multirow{7}{*}{ Local de atuação } & Bloco cirúrgico & 5 & 14,70 & \\
\hline & Coordenação & 4 & 11,80 & \\
\hline & Setor cardiovascular & 4 & 11,80 & \\
\hline & Setor oncológico & 8 & 23,50 & \\
\hline & Setor nefrológico & 4 & 11,80 & \\
\hline & UTI & 4 & 11,80 & \\
\hline & Urgência & 5 & 14,70 & \\
\hline \multirow{4}{*}{$\begin{array}{c}\text { Já atendeu usuários de próteses } \\
\text { dentárias removíveis? }\end{array}$} & Sim & 30 & 88,20 & \multirow{3}{*}{34} \\
\hline & Não & 3 & 8,80 & \\
\hline & Não prestei atenção & 1 & 2,90 & \\
\hline & Não costuma observar isso & 2 & 5,90 & \\
\hline
\end{tabular}


Manejo com as próteses dentárias removíveis

\begin{tabular}{ccc}
\hline $\begin{array}{c}\text { Permite que o paciente fique com a } \\
\text { prótese sempre }\end{array}$ & 5 & 14,70 \\
\hline $\begin{array}{c}\text { Remove e entrega aos familiares, para } \\
\text { que eles se responsabilizem }\end{array}$ & 15 & 44,10 \\
\hline $\begin{array}{c}\text { Orienta a higienização e } \\
\text { acondicionamento das próteses }\end{array}$ & 12 & 35,30 \\
\hline
\end{tabular}

34

Já recebeu treinamento a respeito do manejo com próteses dentárias?

$$
\text { Sim }
$$

3

8,80

91,20

\begin{tabular}{|c|c|c|c|c|}
\hline \multirow{2}{*}{$\begin{array}{l}\text { Acha interessante receber } \\
\text { treinamento a respeito do manejo } \\
\text { com próteses dentárias? }\end{array}$} & Sim & 33 & 97,10 & \multirow[t]{2}{*}{34} \\
\hline & Não & 1 & 2,90 & \\
\hline \multirow{2}{*}{$\begin{array}{c}\text { Realiza a higienização das próteses } \\
\text { dos pacientes internados? }\end{array}$} & Sim & 3 & 8,80 & \multirow[t]{2}{*}{34} \\
\hline & Não & 31 & 91,20 & \\
\hline \multirow{5}{*}{$\begin{array}{c}\text { Quais materiais utiliza para } \\
\text { higienizar as próteses dos } \\
\text { pacientes? }\end{array}$} & Não respondeu & 30 & 88,20 & \multirow{5}{*}{34} \\
\hline & Escova de dente & 1 & 2,90 & \\
\hline & Gaze & 1 & 2,90 & \\
\hline & Hipoclorito de sódio & 1 & 2,90 & \\
\hline & Outro (não especificou) & 1 & 2,90 & \\
\hline \multirow{13}{*}{$\begin{array}{l}\text { Caso você fosse orientar } \\
\text { a higiene das próteses de um } \\
\text { paciente consciente internado } \\
\text { ou do seu acompanhante, quais } \\
\text { os materiais que você } \\
\text { indicaria? }\end{array}$} & Escova & 5 & 14,70 & \multirow{13}{*}{34} \\
\hline & Enxaguante bucal & 3 & 8,80 & \\
\hline & Sabão neutro & 1 & 2,90 & \\
\hline & Hipoclorito de sódio & 2 & 5,90 & \\
\hline & Escova e creme dental & 6 & 17,60 & \\
\hline & Escova e enxaguante & 2 & 5,90 & \\
\hline & Escova, creme dental e enxaguante & 8 & 23,50 & \\
\hline & Escova, enxaguante e hipoclorito & 1 & 2,90 & \\
\hline & Escova, creme dental e sabão & 1 & 2,90 & \\
\hline & Escova e sabão neutro & 1 & 2,90 & \\
\hline & Escova e hipoclorito & 2 & 5,90 & \\
\hline & Escova, creme dental e hipoclorito & 1 & 2,90 & \\
\hline & Escova, enxaguante e sabão & 1 & 2,90 & \\
\hline \multirow{2}{*}{$\begin{array}{c}\text { Costuma solicitar a } \\
\text { remoção de próteses dentárias } \\
\text { removíveis de pacientes } \\
\text { internados? }\end{array}$} & Sim & 25 & 73,50 & \multirow[t]{2}{*}{34} \\
\hline & Não & 9 & 26,50 & \\
\hline \multirow{5}{*}{$\begin{array}{l}\text { Quando você pede para } \\
\text { ele guardar? }\end{array}$} & Não respondeu & 9 & 26,50 & \multirow{5}{*}{34} \\
\hline & $\begin{array}{c}\text { Sempre, só botar quando for comer ou } \\
\text { receber visita }\end{array}$ & 17 & 50,00 & \\
\hline & Quando for dormir & 6 & 17,6 & \\
\hline & Só quando encaminha para UTI & 1 & 2,90 & \\
\hline & Depende da gravidade do quadro & 1 & 2,90 & \\
\hline \multirow{5}{*}{$\begin{array}{l}\text { Qual o local que você } \\
\text { costuma acondicionar } \\
\text { (guardar) ou que } \\
\text { acondicionaria as próteses } \\
\text { dentárias removíveis dos } \\
\text { pacientes? }\end{array}$} & $\begin{array}{c}\text { Em uma sacola etiquetada com os } \\
\text { demais pertences }\end{array}$ & 11 & 32,40 & \multirow{5}{*}{34} \\
\hline & Enrolada numa gaze & 10 & 29,40 & \\
\hline & Enrolada num guardanapo & 1 & 2,90 & \\
\hline & Copo com água & 5 & 14,70 & \\
\hline & $\begin{array}{c}\text { Copo com água com hipoclorito de } \\
\text { sódio }\end{array}$ & 3 & 8,80 & \\
\hline
\end{tabular}




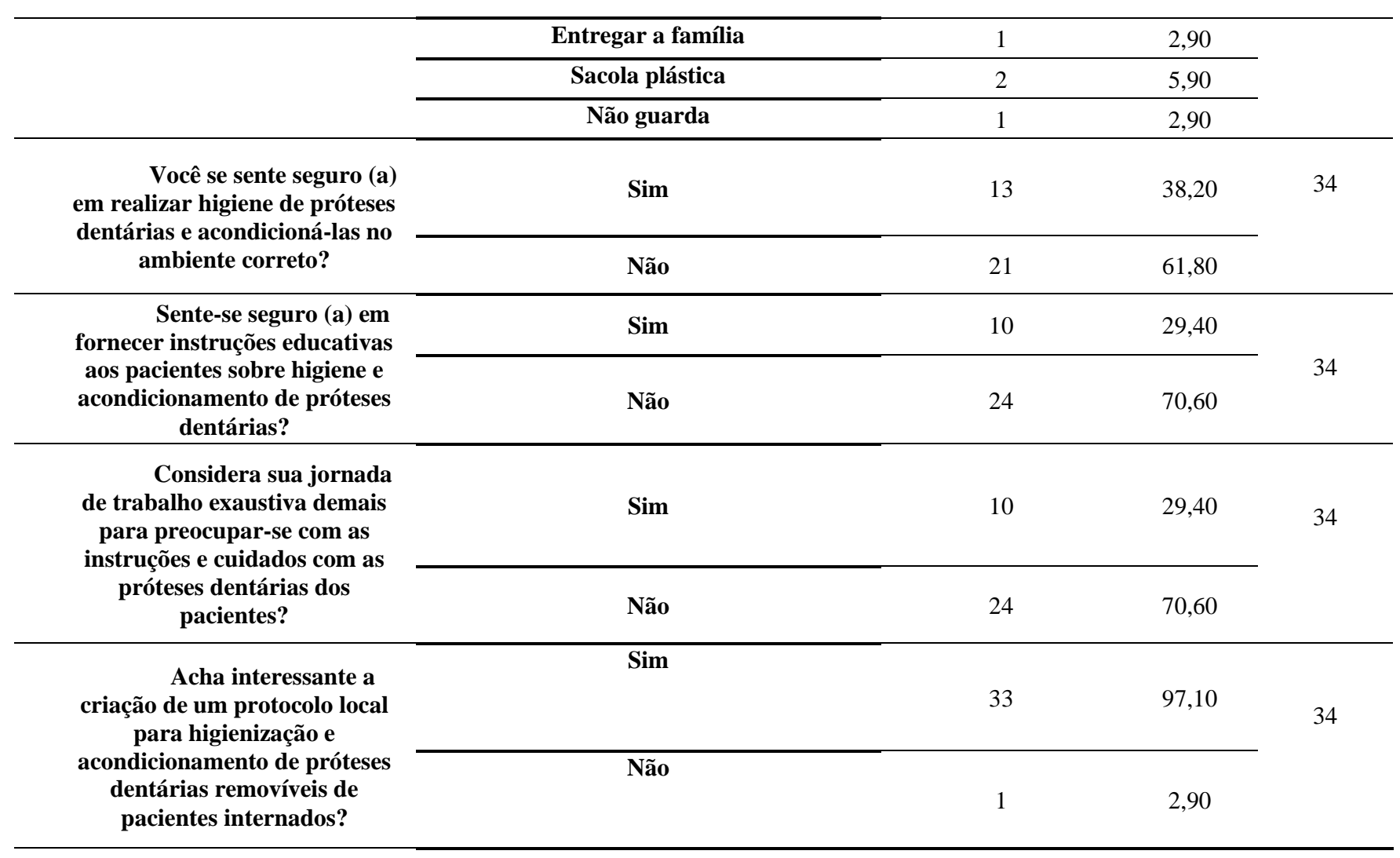

Fonte: Dados da pesquisa.

Para análises associativas, foram categorizadas as variáveis relacionadas aos materiais indicados para higiene das próteses e a forma de acondicionamento dos dispositivos. Diante disso, foi observada associação entre as variáveis "resposta errada" para indicação dos materiais de higiene e não ter recebido treinamento prévio $(\mathrm{RR}=10,40 ; \mathrm{IC}=0,78-137,83 ; \mathrm{p}=0,039)$. Além disso, foi identificada associação entre ter respondido errado e achar interessante a criação de um protocolo local para higienização e acondicionamento de próteses ( $\mathrm{RR}=0,18 ; \mathrm{IC}=0,08-0,37 ; \mathrm{p}=0,046)$. Ou seja, as enfermeiras sentiram dificuldade em responder de forma correta, por não terem recebido nenhuma ou pouca instrução prévia a respeito do assunto, e sentem a necessidade da criação de um protocolo operacional padrão para melhor atender aos pacientes internados (Tabela 2). 
Tabela 2 - Associação entre não ter recebido treinamento prévio a respeito de higiene e acondicionamento de próteses e ter indicado de forma errada.

\begin{tabular}{|c|c|c|c|c|c|c|}
\hline \multirow{4}{*}{$\begin{array}{c}\text { Recebimento de } \\
\text { treinamento prévio } \\
\text { sobre higiene e } \\
\text { acondicionamento de } \\
\text { próteses }\end{array}$} & & \multicolumn{2}{|c|}{$\begin{array}{l}\text { Materiais indicados para higiene } \\
\text { de próteses }\end{array}$} & \multirow{3}{*}{$\begin{array}{c}\text { p-valor } \\
0,039^{*}\end{array}$} & \multirow{3}{*}{$\begin{array}{c}\mathbf{R R} \\
10,40\end{array}$} & \multirow{3}{*}{$\begin{array}{c}\text { IC }(\mathbf{9 5} \%) \\
0,78-137,83\end{array}$} \\
\hline & & $\begin{array}{c}\text { Resposta } \\
\text { correta }\end{array}$ & Resposta errada & & & \\
\hline & Sim & 2 & 1 & & & \\
\hline & Não & 5 & 26 & & & \\
\hline \multirow{2}{*}{$\begin{array}{c}\text { Criação de um } \\
\text { protocolo local para } \\
\text { higienização e } \\
\text { acondicionamento de } \\
\text { próteses }\end{array}$} & Sim & 6 & 27 & \multirow[t]{2}{*}{$0,046^{*}$} & \multirow[t]{2}{*}{0,18} & \multirow[t]{2}{*}{$0,08-0,37$} \\
\hline & Não & 1 & 0 & & & \\
\hline
\end{tabular}

Teste Exato de Fisher; *p<0,05; RR=risco relativo; IC=intervalo de confiança de 95\%. Fonte: Dados da pesquisa.

\section{Discussão}

Múltiplas atribuições são direcionadas à equipe de enfermagem frente aos pacientes hospitalizados, dentre elas, a higienização corporal do paciente, incluindo a higiene bucal (Palm \& Azambuja, 2019). Porém, para realização desses cuidados é necessário que haja conhecimento prévio.

Apesar da equipe de enfermagem possuir um papel importante sobre os resultados em saúde bucal, essa prática ainda é muito limitada nos hospitais (Coker et al., 2016). Na maioria dos casos, a equipe não possui conhecimento suficiente para realização dos cuidados bucais e sente-se limitada para adentrar numa área tão minuciosa (Weintraub et al., 2018). A falta de conhecimento a respeito de protocolos, princípios e métodos de higiene bucal contribui para a negligência por parte dos enfermeiros, podendo resultar em agravos ao paciente, como o deslocamento do biofilme para orofaringe e disseminação dos microrganismos para os demais sistemas, principalmente para o respiratório. Isso também se aplica às instruções com relação aos cuidados com as próteses dentárias que esses profissionais poderiam dar aos pacientes (Coker et al., 2016; Vilela, Ferreira, Santos, Rezende, 2015; Fonseca et al., 2019).

Portanto, faz-se necessária a intervenção do CD, a fim de educar e treinar a equipe de enfermagem para uma correta e segura realização dos cuidados de higiene bucal (Weintraub et al., 2018).

Sobre a desinfecção das próteses, Papadiochou e Polyzois (2017) mostram que existem alguns métodos: mecânico, químico, irradiação ou combinado. O mecânico é realizado através da escovação ou utilização de ultrassom. Métodos de irradiação ocorrem através de micro-ondas ou terapias fotodinâmicas (laser de baixa potência). Métodos químicos são executados através da imersão da prótese em algumas substâncias, como: hipoclorito, peróxidos, enzimas, ácidos e enxaguantes bucais. Porém, algumas substâncias podem ser facilmente ingeridas pelo paciente, principalmente, tratando-se de idosos, por apresentarem semelhanças com os medicamentos consumidos diariamente, podendo causar danos à saúde do paciente (Doshi \& Jones, 2019). Em seu estudo, Papadiochou e Polyzois (2017) afirmam que, o método ideal seria aquele que apresentasse ação fungicida e bactericida, além de manter a dimensão, estabilidade e cor da prótese.

No que diz respeito ao tipo de material indicado pelas enfermeiras do presente estudo, para realização da limpeza das próteses dentárias, prevaleceu: escova, creme dental e enxaguante, que de acordo com Cakan et al. (2015) a escovação é o mais indicado, por ser o mais simples e de menor custo. Todavia, quando combinado ao método químico, apresenta resultados mais satisfatórios, devido à redução efetiva na quantidade de microrganismos.

A remoção da prótese dentária removível dos pacientes internos no HSVP é solicitada pelas enfermeiras e indicado usar apenas quando forem receber visitas ou para alimentar-se. Estudos prévios indicam que a prótese dentária seja removida durante 
o sono noturno, objetivando a redução da proliferação de microrganismos patogênicos no ambiente bucal e na prótese dentária do paciente. Em adição, a remoção desse dispositivo durante o sono, promove o relaxamento dos tecidos que são comprimidos pelo uso contínuo da mesma (Verhaeghe et al., 2019; Oliveira, Martins \& Vasconcelos, 2020).

Por outro lado, um estudo realizado por Mann e Doshi (2017) aponta que a não utilização da prótese dentária pode influenciar no bem-estar do paciente, em consequência de reduzir sua autoestima e prejudicar a interação social. Geralmente, a remoção da prótese traz consigo problemas na fonação e estética, afetando a qualidade de vida do usuário (Fonseca et al., 2019). No entanto, no ambiente hospitalar essa indicação de remoção faz-se necessária, para que o paciente evite riscos de infecções oportunistas e/ou deglutição ou broncoaspiração dos dispositivos (Palm \& Azambuja, 2019).

No presente estudo, a forma mais frequente de acondicionamento das peças protéticas foram em sacolas identificadas com os demais pertences, sendo, portanto, contrária aos achados de Verhaeghe et al., (2019); Fonseca et al., (2019), que afirmam que o acondicionamento noturno em ambiente úmido é indicado para evitar proliferações microbianas e instalação de infecções oportunistas, bem como para evitar mudanças estruturais nas propriedades dos materiais que compõem as próteses, juntamente com as informações pessoais do paciente. No ambiente hospitalar, essas práticas não são padronizadas, nem feitos corriqueiramente. A ausência de materiais para realização de higiene e acondicionamento da mesma contribuem para esta falta de cuidado (Fonseca et al., 2019).

Numa pesquisa realizada por Coker et al. (2016) cita que: Os enfermeiros alegam excesso de atribuições e por este motivo a higiene bucal acaba sendo negligenciada. Entretanto, especificamente neste estudo realizado no HSVP, as enfermeiras alegaram não possuir uma jornada exaustiva de trabalho, e acham interessante a criação e implementação de protocolos de higienização e acondicionamento de próteses dentárias, para que possam executá-los com responsabilidade e excelência. A falta de protocolo/hábito pode estar ligada a negligência dessas atribuições.

Este estudo apresentou algumas limitações, como a realização da coleta de dados em ambiente hospitalar durante a pandemia do SARS-CoV-2 e resistência de algumas das enfermeiras para responder o questionário durante seu horário de expediente, a partir da qual tivemos duas recusas. O número limitado de enfermeiras, configurando uma amostra pequena, também pode ser referido como uma limitação. No entanto, tais limitações não foram capazes de afetar o desfecho final da pesquisa, uma vez que, os protocolos de biossegurança exigidos pela Agência Nacional de Vigilância Sanitária (ANVISA) foram seguidos rigorosamente durante toda pesquisa no ambiente hospitalar. Além disso, o questionário foi aplicado de acordo com a disponibilidade de cada profissional. Mesmo com um número amostral pequeno, os resultados são representativos e os dados podem servir de base para realização de estudos futuros.

Esse tipo de abordagem é essencial para identificar dificuldades encontradas pela equipe de enfermagem, ao se depararem com pacientes usuários de prótese dentária no ambiente hospitalar. Deste modo, é interessante que essa metodologia seja aplicada em outros hospitais, juntamente com uma metodologia intervencional, para que haja melhorias na rotina hospitalar e o treinamento educativo da equipe seja efetivo.

Observando a deficiência de conhecimento da equipe sobre higienização e acondicionamento de prótese dentária, sugere-se que antes de iniciar os atendimentos de usuários de próteses dentárias hospitalizados, os enfermeiros passem por uma capacitação, com ênfase na importância de manter a higienização bucal, armazenamento e limpeza das próteses de seus pacientes, bem como, incentivá-los a realizar a própria limpeza de seus dispositivos, quando se fizer possível e necessário.

\section{Considerações Finais}

Diante do exposto, pode-se concluir que devido à falta de instrução durante a formação e treinamentos no ambiente hospitalar por um cirurgião-dentista capacitado, a equipe de enfermagem não possui conhecimento suficiente e adequado, sobre 
os cuidados com as próteses dentárias dos pacientes (higienização e acondicionamento). As enfermeiras não se sentem confortáveis em ministrar orientações aos pacientes sobre a higienização e armazenamento desses dispositivos. Também não há um padrão de acondicionamento de próteses seguido pelas enfermeiras do hospital, muitas vezes armazenadas de forma errônea (envolta em uma gaze, envolta em um guardanapo, copo com água, copo com água e hipoclorito, sacola plástica), o que facilita a perda desse objeto.

É evidente a necessidade de padronização e implementação de protocolos de higienização e acondicionamento de prótese dentárias removíveis no ambiente hospitalar, bem como o treinamento da equipe por um cirurgião-dentista, a fim de ministrar informações que capacitem estes profissionais realizarem orientações sobre higienização e acondicionamento adequado das próteses dentárias com propriedade e segurança.

\section{Referências}

Amaral, C. O. F., Belon, L. M. R., Silva, E. A., Nadai, A., Amaral Filho, M. S. P., \& Straioto, F. G. (2018). The importance of hospital dentistry: oral health status in hospitalized patients. REV. Gaúch Odontol, 66(1), 35-41.

Aranega, A. M., Bassi, A. P. F., Ponzoni, D., Wayama, M. T., Esteves, J. C., \& Garcia Junior, I. R. (2012). Qual a importância da Odontologia Hospitalar? Revista Brasileira de Odontologia, 69(1), 90-93.

Axe, A. S., Varghese, R., Bosma, M., Kitson, N., \& Bradshaw, D. J. (2015). Dental health professional recommendation and consumer habits in denture cleansing. The Journal of Prosthetic Dentistry, 115(2), 183.

Baba, Y., Sato, Y., Owada, G., \& Minakuchi, S. (2018). Effectiveness of a combination denture-cleaning method versus a mechanical method: comparison of denture cleanliness, patient satisfaction, and oral health-related quality of life. Journal of Prosthodontic Research. 62(3), 353-358.

Blum, D. F. C., Silva, J. A. S., Baeder, F. M.,\& Bona, A. D. (2018). A atuação da odontologia em unidades de terapia intensiva no Brasil. Rev. Bras Ter Intensiva, 30(3), 327-332.

Cakan, U., Yuzbasioglu, E., Kurt, H., Kara, H. B., Turunç, R., Akbulut, A., \& Aydin, K. C. (2015). Assessment of hygiene habits and attitudes among removable partial denture weares in a university hospital. Nigerian Journal or Clinical Practice, 18(4), 511-5.

Coker, E., Ploeg, J., Kaasalainen, S., \& Carter, N. (2016). Nurse's oral hygiene care praticces with hospitalized older adults in postacute settings. International Journal of Older People Nursing, 12(1).

Conselho Regional de Enfermagem. (2018). Código de Ética de Enfermagem. São Paulo. Recuperado de https://portal.coren-sp.gov.br/wpcontent/uploads/2018/11/Codigo-de-etica.pdf.

Dantas, A. P. F. M., Consani, R. L. X., Sardi, J. C. O., Mesquita, M. F., Silva, M. C. V. S., \& Sinhoreti, M. A.C. (2014). Biofilm Formation in Denture Base Acrylic Resins and Disinfection Method Using Microwave. Journal of Research and Practice in Dentistry.

Doshi, M., \& Jones, V. (2019). Denture hygiene Safety issues with denture care. British Dental Journal, 226(1).

Fonseca, E. O. S., Pedreira, L. C., Gomes, N. P., Amaral, J. B., Virgens, I. R., \& Santos, F. C. (2019). O cuidado de enfermagem no acondicionamento da prótese dentária de idosos hospitalizados. Acta Paul Enferm, 32(4), 442-8.

Gonçalves, L. F. F., Silva Neto, D. R., Bonan, R. F., Carlo, H. L., \& Batista, A. U. D. (2011). Higienização de próteses totais e parciais removíveis. Revista Brasileira de Ciência da Saúde, 15(1), 87-94.

Mann, J., \& Doshi, M. (2017). An investigation into denture loss in hospitals in Kent, Surrey and Sussex. British Dental Journal, $223(3)$, 435-438.

Mattevi, G. S., Melo, A. L. S. F., Scannapieco, F. A., \& Carcereri, D. L. (2018). The dentist's role in southern Brazilian teaching hospitals: a grounded theory study. Journal of Dental Education, 82(6), 636-643.

Oliveira, M. D. S., Martins, T. G., \& Vasconcelos, G. L. L. (2020). Influência da higienização das próteses removíveis na saúde bucal e sistêmica dos pacientes. Jnt-Facit Business And Technology Journal, 3(19), 96-108.

Palm, F. C., \& Azambuja, N. G. (2019). Condições de próteses dentárias em pacientes hospitalizados: sugestão de protocolo. (Monografia). Universidade de Santa Cruz do Sul- UNISC. Santa Cruz do Sul-RS.

Papadiochou, S., \& Polyzois, G. (2017). Hygiene practices in removable prosthodontics: a systematic review. International Journal of Dental Hygiene, 16(2), $179-201$.

Rocha, A. L., \& Ferreira, E. (2014). Odontologia hospitalar: a atuação do cirurgião dentista em equipe multiprofissional na atenção terciária. Arq Odontol, 50(4), 154-160.

Shwe, P. S., Ward, S. A., Thein, P. M., \& Junckerstorff, R. (2019). Frailty, oral health and nutrition in geriatrics inpatients: a cross-sectional study. Gerodontology, 36(3), 223-228. 
Research, Society and Development, v. 10, n. 10, e475101018471, 2021 (CC BY 4.0) | ISSN 2525-3409 | DOI: http://dx.doi.org/10.33448/rsd-v10i10.18471

Van Noort, H. H. J., Witteman, B. J. M., Hertog-Voortman, R., Everaars, B., Vermeulen, H., \& Waal, G. H. (2019). A context analysis on how care is delivered in hospitalized patients: a mixed-methods study. Journal of Clinical Nursing, 29, 1991-2003.

Verhaeghe, T. V., Wyatt, C. C., \& Mostafa, N. Z. (2019). The effect of overnight storage conditions on complete denture colonization by Candida albicans and dimensional stability: A systematic review. The Journal of Prosthetic Dentistry, 124(2), 176-182.

Vilela, M. C. N., Ferreira, G. Z., Santos, P. S. S., \& Rezende, N. P. N. (2015). Cuidados bucais e pneumonia nosocomial: revisão sistemática. Einstein, 13(2), 290-6.

Weintraub, J. A., Zimmerman, S., Ward, K., Wretman, C., Sloane, P. D., Stearns, S. C., Poole, P., \& Preisser, J. S. (2018). Improving nursing home residents' oral hygiene: results of a cluster randomized intervention trial. Jamda, 19(12), 1086-1091. 